\title{
Carotid Revascularization with and without the Use of an Embolic Protection Device: A Single-Center Experience from Pakistan
}

\author{
Qasim Bashira, b Ammad Anwar Baig ${ }^{c}$ \\ aDepartment of Neurointervention, Bahria Town Hospital, Lahore, Pakistan; ${ }^{b}$ Department \\ of Clinical and Interventional Neurology, CMH Lahore Medical College, Lahore, Pakistan; \\ ${ }^{\mathrm{c}} \mathrm{CMH}$ Lahore Medical College, Lahore, Pakistan
}

\section{Keywords}

Embolic protection device $\cdot$ Stroke $\cdot$ Carotid artery stenting $\cdot$ Intervention

\begin{abstract}
Background: To assess the safety and clinical efficacy of carotid artery stenting with and without an embolic protection device (EPD) in both symptomatic and asymptomatic carotid disease cases. Methods: Retrospective data of 55 symptomatic ( $\geq 50 \%$ occlusion by digital subtraction angiography [DSA], $\geq 70 \%$ by ultrasound, computed tomography angiography [CTA], and magnetic resonance angiography [MRA]) and asymptomatic ( $\geq 60 \%$ by DSA, $\geq 70 \%$ by ultrasound, $\geq 80 \%$ by CTA and MRA) carotid disease cases undergoing carotid stenting/angioplasty revascularization from February 2014 to October 2017 was reviewed. All symptomatic patients either experienced recurrent transient ischemic attacks or one or more stroke attacks. An EPD protocol was designed for its selective use based on plaque morphologies and working diameters. The primary end points at 30 days of follow-up were a periprocedural incidence of any stroke, myocardial infarction or death, and ipsilateral stroke during the follow-up period. Results: Of the 55 cases, 39 were males and 16 females; mean age was 64.8 years. Fiftyone patients (92.7\%) were symptomatic, with a mean stenosis of $80.1 \%$. EPD was used in only 11 cases (20\%). Minor stroke rate during the first 30 postoperative days was $1.8 \%$ (1 case) with EPD; no myocardial infarction or mortality. No stroke occurred during the median 1.5 years' follow-up. Conclusion: Based on our single-center experience and findings of a relatively
\end{abstract}

The authors would like to highlight the fact that a part of this paper was accepted and presented at the 2016 Annual SVIN conference held in Bronx, New York, USA, entitled "Initial Experience of Carotid Revascularization without Embolic Protection Device." 
small sample size, carotid revascularization with stenting and angioplasty without EPD in experienced hands was found to be safe and efficacious. In addition, it proves cost-effective for patients by limiting the use of unnecessary disposables. These results are comparable to those reported in major trials and are well within the complication thresholds suggested in current guidelines. These results also show promise and illustrate the need for a larger, randomized controlled trial in order to thoroughly address this aspect of carotid revascularization.

(C) 2018 S. Karger AG, Basel

\section{Introduction}

Stroke is the second most common cause of death globally and the leading cause of serious long-term disability in the United States [1]. A number of studies have established carotid stenosis as a significant contributor to stroke [2,3]. Carotid endarterectomy (CEA) and carotid artery stenting (CAS) are the surgical and nonsurgical methods of stroke prevention, respectively.

The efficacy and safety of CAS has been tested through several randomized and nonrandomized trials but the debate continues as to whether CAS is an absolute alternative to CEA. Amongst these studies, the most recent, Carotid Revascularization Endarterectomy versus Stenting Trial (CREST), and the Protected Carotid-Artery Stenting versus Endarterectomy in High-Risk Patients (SAPPHIRE) have highlighted the at-par potential of CAS with CEA [4-6]. Long-term results of over 10 years of follow-up found no significant difference between patients who underwent CAS compared to those who underwent CEA [5]. Both procedures were found to be equally durable in the long term, with infrequent restenosis after either procedure. Following these trials, CAS has emerged as a reliable alternative revascularization technique for extracranial carotid stenosis.

In order to reduce embolic stroke during CAS procedures, an embolic protection device (EPD) has been advocated for use [7, 8]. The use of an EPD adds to manipulation and hence apart from adding to the cost, it increases procedural risks [9]. Maynar et al. [10], and most recently Binning et al. [11] in their cases of CAS without EPD, showed that the placement of a stent before or after balloon dilation was both safe and efficacious.

In developing countries, health-related costs are primarily borne by the patients or their family members and neuroendovascular disposables significantly add to the total cost. In addition to reporting carotid revascularization data from a developing country like Pakistan, we aim to establish, in all cases of carotid stenosis, the role of operator skills rather than the nature or complexity of stenosis as a contributor towards the outcome of the procedure. In addition, we aim to verify the results of other studies of similar nature but from developed countries. We report our initial experience of 55 consecutive CAS procedures both with and without the use of an EPD.

\section{Methodology}

\section{Patient Selection}

This is a single-center, retrospective study of 55 consecutive CAS cases from February 2014 to August 2017, both symptomatic and asymptomatic. The study was approved by the Institutional Review Board and Ethics Committee. All procedures were performed by a single operator, who met the criteria set by the CREST investigators and the guidelines established by the American Heart Association (AHA) $[4,5,12]$. The patients were labeled as symptomatic if having $\geq 50 \%$ occlusion by digital subtraction angiography (DSA), and $\geq 70 \%$ by ultrasound, computed tomography angiography (CTA), or magnetic resonance angiography (MRA), and as asymptomatic if having $\geq 60 \%$ occlusion by DSA, $\geq 70 \%$ by ultrasound, and $\geq 80 \%$ by CTA or MRA. 
Bashirand Baig: Carotid Revascularization with and without EPD
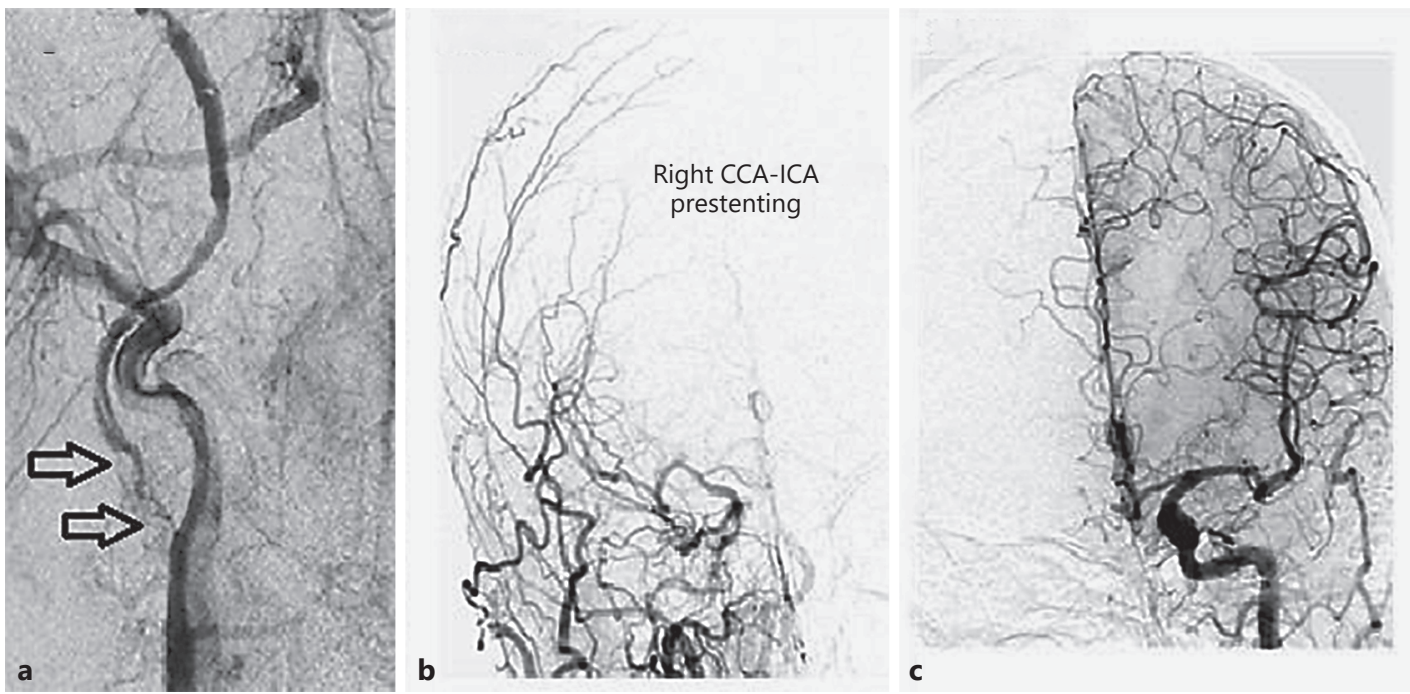

Fig. 1. a-c Case 1 - 65 years old, male, with right middle cerebral artery stroke. Critical right carotid bulb stenosis (a) treated without embolic protection device. Prestenting intracranial angiography shows noticeable delay (b). No cross-filling of the right hemisphere from the left anterior communicating artery is seen (c). $\mathbf{d}-\mathbf{g}$ Case 1 continued. f Poststenting image. g Noticeable intracranial flow improvement seen following stenting. Compare with prestenting image (d, e).
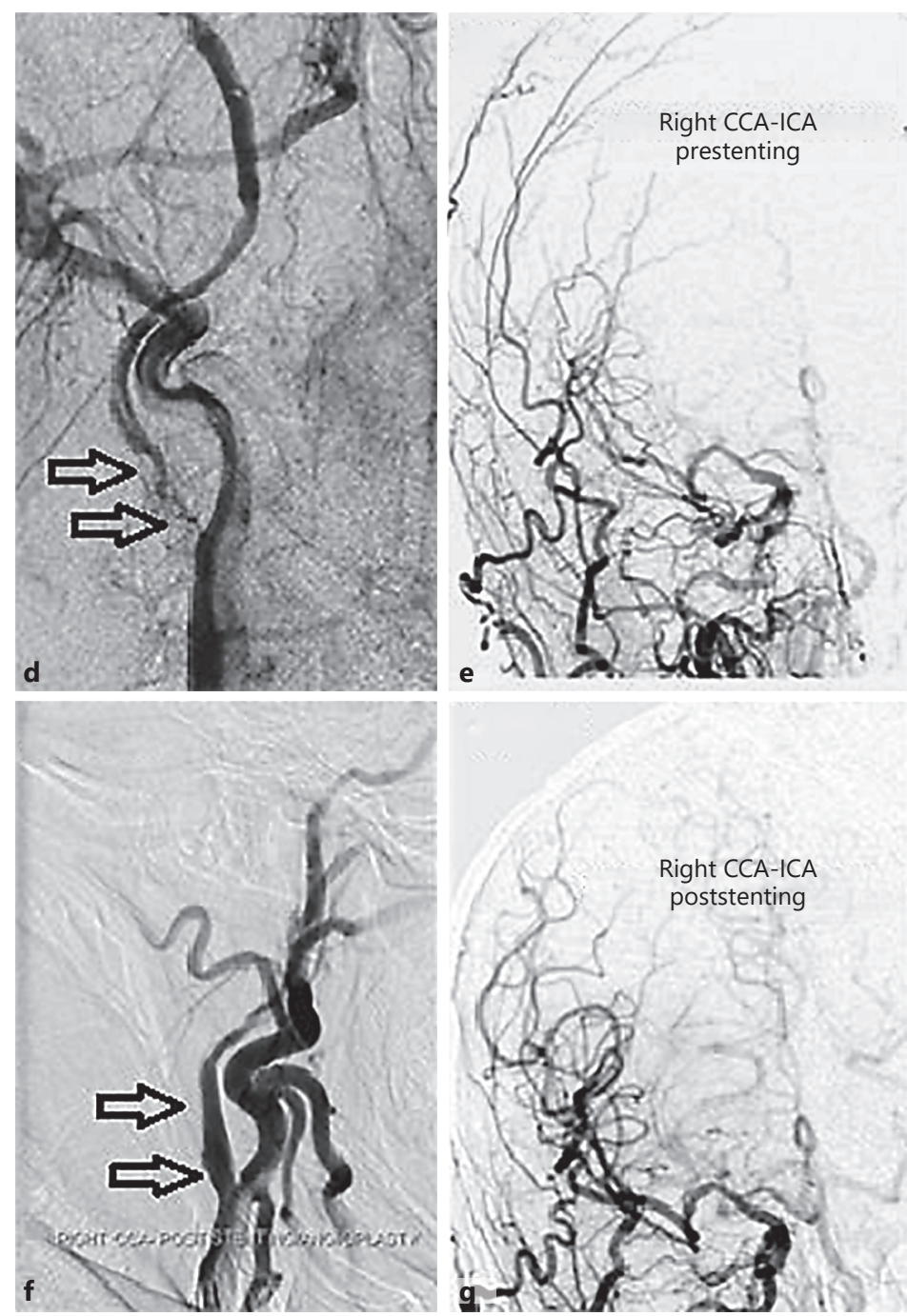
All symptomatic patients had either suffered a transient ischemic attack or a minor or major disabling stroke prior to being treated for stenosis. They were treated with the best medical management and followed up post-procedure as per the CREST criteria [4]. Patients were followed for a period of 30 days during which time the primary end points, being a periprocedural incidence of any severity stroke (unilateral or bilateral), myocardial infarction or death, and ipsilateral stroke during the follow-up period, were recorded. The mean follow-up period was 1.5 years, with the longest period extending up to 3 years.

\section{Angiographic Characteristics}

Basic demographics including age, gender, and symptomatic status were recorded. Angiographic characteristics were obtained from pre- and post-procedural angiograms using DSA. Characteristics included arterial lesion lengths, percent diameter stenosis, plaque morphology, and nature of the lesion as being either contiguous or sequential/remote. Since plaque morphology was an important criterion in our protocol designed for the selective use of EPD, using the classification proposed by Lovett el al. [13], these were typed as ulcerated, smooth or irregular. All plaque morphologies were recorded before stenting using DSA.

Accurate measurements were made, in millimeters, for the lesion lengths with the stenotic segment defined as that extending from the proximal to the distal point of maximum stenosis. Percent diameter stenosis were calculated as per NASCET criteria, whereby categorization was done for patients having mild $(<50 \%)$, moderate $(50-70 \%)$ or severe $(>70 \%)$ stenosis [14]. Once all the lesion measurements were recorded, joint arterial characteristics were identified and patient classification was done based on any number of combination of these. Apart from these, pre- and post-stent balloon angioplasties were also performed and recorded for individual patients.

\section{Procedure}

All stenting procedures were performed according to the guidelines agreed upon by the American Heart Association Task Force on practice guidelines and the American Stroke Association in the 2011 joint statement [12]. Procedural consent was obtained after describing procedural risks, benefits, and alternatives. Patients were maintained on dual antiplatelet therapy for at least 1 week prior to the procedure on a dosage regimen of $75 \mathrm{mg}$ aspirin and $75 \mathrm{mg}$ clopidogrel once daily. This course of treatment was maintained for at least 3 months (90 days) after the procedure. After administration of local anesthesia, the femoral artery was punctured and an 8-Fr short catheter sheath was placed in the artery. Then, a 6-Fr 90-cm sheath was advanced over a 5-Fr guide catheter using the parallax technique. A preshaped 0.014-mm microwire was carefully advanced and its distal tip positioned beyond the petrous internal carotid artery segment. In all cases, our objective was to achieve an at least 2-mm working diameter at the most stenotic point for smooth delivery of the stent. Prestent angioplasty hence was performed using a coronary compliant balloon catheter with length and diameter tailored to the stenotic area measurements (Fig. 1). We preferred, if possible, to perform prestent angioplasty only once followed by a quick delivery of the carotid stent. As a practice, we do not use contrast injector for CAS procedures. A self-expandable open-cell stent system was used in all cases (except in 2 cases, where a Boston Scientific Wallstent was used). Although the size of the stent varied according to lesion length, the sizes most commonly used were $6-8 \times$ $40,6-8 \times 30$, and $7 \times 30 \mathrm{~mm}$. All cases, except 3 , required a single-stent placement to completely cover the stenotic segment.

\section{EPD Protocol and Follow-Up}

The use of an EPD was separately recorded. Distal filter type of EPD was used where deemed necessary. EPDs used during our study included: Abbott RX Accunet Embolic Protection Device (8 cases), Boston Scientific FilterWire EZ Embolic Protection System (2 cases), and Cordis RX Angioguard Embolic Protection Device (1 case). Plaque morphology and working diameters were considered to be the foremost criteria in the selective use of EPD. EPD was used in cases where the plaques were found to have one or more than one of the following characteristics: (1) unstable/mobile nature (Fig. 2), (2) circumferential severe wall ulcerations, or (3) irregular morphology. Although $60 \%$ of our cases had ulcerations, only $20 \%$ received EPD treatment because a safe and workable diameter was attained (through prestent angioplasties) to the operators' satisfaction (Fig. 3, 4). The plaques were found to be ulcerated in a vast majority of cases where EPD was used. Poststenting control angiographies were performed in all cases to check for anterograde filling defects, residual stenosis or additional occlusions. 
Fig. 2. Case 2 - 42 years old, male, with recurrent left middle cerebral artery territory infarcts while on best medical therapy. Note a mobile thrombus (arrow) extending from the bulb along the proximal cervical left internal carotid artery (a). Left carotid stenting performed using an embolic protection device (b).
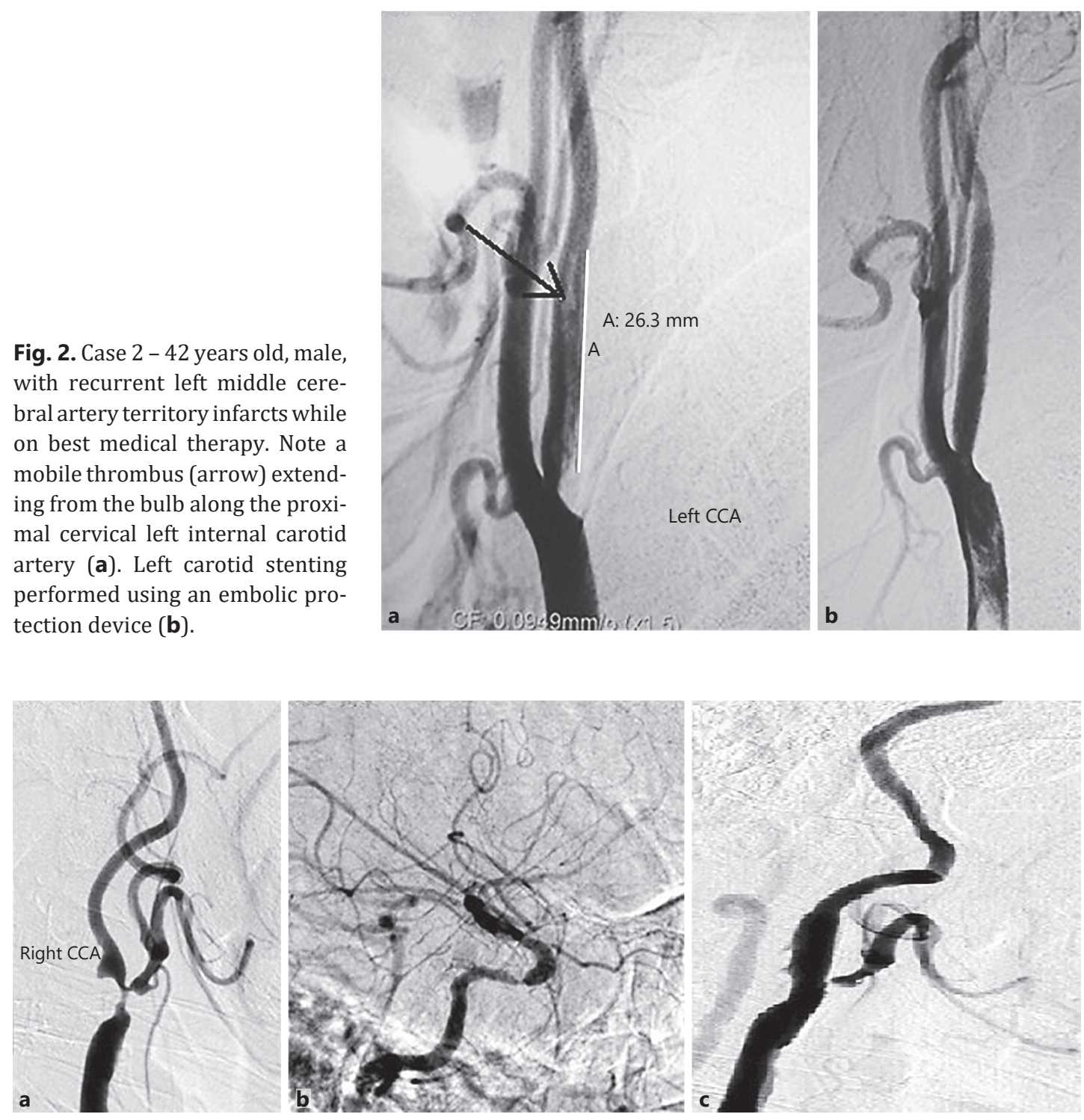

Fig. 3. Case 3 - 76 years old, male, with right middle cerebral artery stroke with right carotid bulb ulcerated stenosis (a). Note on lateral projection, intracranial cavernous internal carotid artery tandem stenosis (b). Poststenting image without embolic protection device (c).

Patients' follow-up, outside of the periprocedural period, for complications such as stroke or in-stent stenosis was performed at 1, 6, and 12 months following the procedure using carotid Doppler ultrasonography and then yearly for up to 4 years. During the follow-up period, a strong emphasis was given to improving vascular risk factors, such as hypertension, diabetes, hyperlipidemia, obesity, and smoking. Primary end point outcomes were recorded during the follow-up period.

\section{Results}

Over the stipulated study period, 55 consecutive carotid stenting procedures were performed on a total of 52 patients ( 3 bilateral stenosis cases) (Table 1). Mean age was 64.8 \pm 10.4 years, ranging from 30 to 81 years. Overall, 39 were male $(70.9 \%)$ and 16 were female 
Fig. 4. Case 4 - 66 years old, female, with symptomatic left carotid bulb stenosis (93-96\%, NASCET). Note incidental doublechambered left distal cervical internal carotid artery (a). Postangioplasty/stenting without embolic protection device (b).
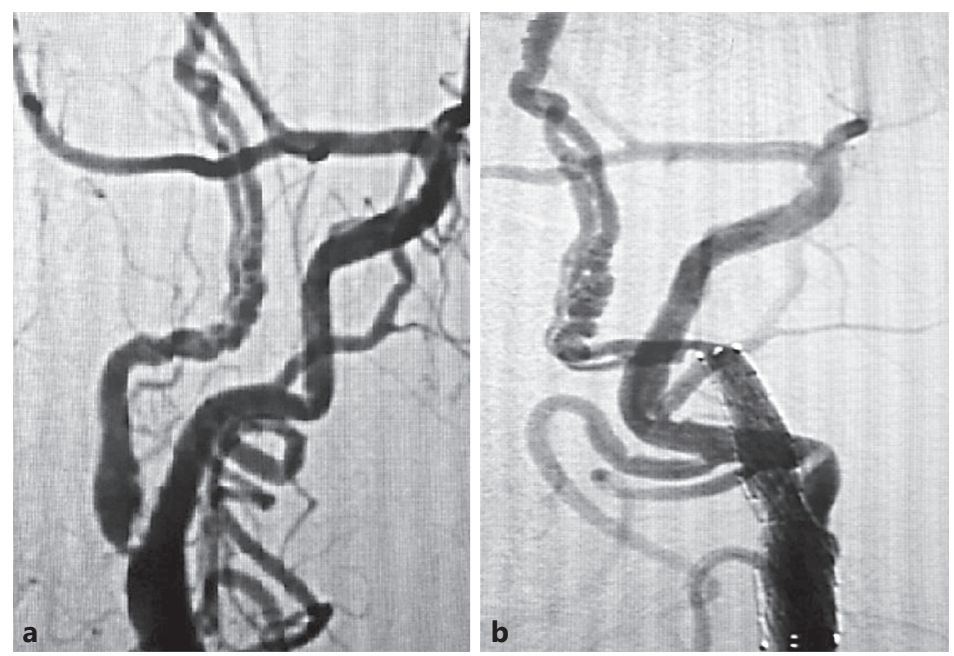

Table 1. Basic demographics and arterial characteristics

\begin{tabular}{lcc}
\hline Factors & $\begin{array}{l}\text { CAS cases } \\
(n=55)\end{array}$ & $\%$ \\
\hline Mean age, years & $64.8 \pm 10.4$ & - \\
Gender & & \\
$\quad$ Male & 39 & 70.9 \\
$\quad$ Females & 16 & 29.1 \\
Symptomatic & 51 & 92.7 \\
Right CAS & 23 & 41.9 \\
Left CAS & 32 & 58.1 \\
Mean lesion length, mm & $25.2 \pm 13.3$ & - \\
Mean percent diameter stenosis & - & 80.1 \\
$\quad$ Severe stenosis $(\geq 70 \%)$ & 44 & 80.0 \\
$\quad$ Moderate stenosis (50-70\%) & 11 & 20.0 \\
Long lesions ( $\geq 24.9$ mm) & 26 & 47.3 \\
Short lesions (<24.9 mm) & 29 & 52.7 \\
Contiguous lesions & 42 & 76.4 \\
Sequential or remote lesion & 13 & 23.6 \\
Narrow ulceration & 33 & 60.0 \\
Contiguous + short & 25 & 45.5 \\
Contiguous + long & 17 & 30.9 \\
Sequential/remote + short & 9 & 16.4 \\
Sequential/remote + long & 4 & 7.3 \\
\hline
\end{tabular}

(29.1\%). Left-sided carotid stenting was performed in 32 cases (58.1\%) and right-sided in 23 (41.9\%).

A vast majority of cases $(92.7 \%)$ were symptomatic while only 4 cases $(7.3 \%)$ were asymptomatic. The mean lesion length recorded was $25.2 \mathrm{~mm}$ ranging from the smallest lesion of $1.6 \mathrm{~mm}$ to the largest at $69.1 \mathrm{~mm}$. Division of these lesions into long and short was based on their relative lengths to a median of $24.9 \mathrm{~mm}$. Almost equal incidence of long (26, $47.3 \%)$ and short lesions $(29,52.7 \%)$ was observed. 59 stents were used on a total of 52 patients (1.13 per patient).

Categorization of patients into severe and moderate cases was done based on percent diameter stenosis, with a mean stenosis of $80.1 \%$ (Table 2). An overwhelming majority (80\%) had severe stenosis and the rest (20\%) had a moderate level of stenosis. Seventy-six percent 
Bashirand Baig: Carotid Revascularization with and without EPD

Table 2. Procedure results

\begin{tabular}{lcc}
\hline Results & $\begin{array}{c}\text { CAS cases } \\
(n=55)\end{array}$ & $\%$ \\
& $11 / 55$ & 20.0 \\
\hline Embolic protection device & $1 / 55$ & 1.8 \\
Mild stroke & $52 / 55$ & 94.5 \\
Total number of angioplasties & $26 / 52$ & 50.0 \\
Both pre- and poststenting & $11 / 52$ & 21.2 \\
Prestenting only & $15 / 52$ & 28.8 \\
Poststenting only & $59(1.13)$ & - \\
Total number of stents used (average) & 5 & - \\
Types of stents used & $25 / 59$ & 42.4 \\
$\quad$ Abbott Acculink & $27 / 59$ & 45.8 \\
ev3 Protégé & $3 / 59$ & 5.1 \\
$\quad$ Boston Scientific Liberté & $2 / 59$ & 3.4 \\
Boston Scientific Wallstent & $2 / 59$ & 3.4 \\
Cordis & & \\
\hline
\end{tabular}

Table 3. EPD data

\begin{tabular}{lcr}
\hline Results & $\begin{array}{l}\text { EPD cases } \\
(n=11)\end{array}$ & $\%$ \\
\hline Severe wall ulcerations & 9 & 81.8 \\
Irregular morphology & 1 & 9.1 \\
Mobile/unstable plaque & 1 & 9.1 \\
Mean percent diameter stenosis & & 78.5 \\
$\quad$ Severe stenosis ( $\geq 70 \%)$ & 10 & 90.9 \\
$\quad$ Moderate stenosis (50-70\%) & 30.6 & 9.1 \\
Mean lesion length (EPD group), mm & & - \\
Lesion type & 6 & 54.5 \\
$\quad$ Contiguous + long & 2 & 18.2 \\
$\quad$ Contiguous + short & 1 & 9.1 \\
$\quad$ Sequential/remote + short & 2 & 18.2 \\
$\quad$ Sequential/remote + long & 11 & 100 \\
Total angioplasties in EPD group & $10 / 11$ & 90.9 \\
Total prestent angioplasties & $7 / 11$ & 63.6 \\
Both pre- and poststent angioplasties & & \\
\hline
\end{tabular}

of these lesions were of contiguous nature, with $24 \%$ sequential lesions. Narrow ulcerative plaques were seen in $60 \%$ of cases.

Amongst the joint arterial characteristics, contiguous plus short lesions were most commonly seen in 25 cases ( $45.5 \%$ ) (Table 2 ) followed closely by contiguous plus long lesions in $18(32.7 \%)$. Balloon angioplasties were performed in 52 cases $(94.5 \%)$ and of these, 26 $(50 \%)$ underwent both pre- and poststent angioplasties (Table 2). The follow-up rate was $100 \%$ for 1.5 years' duration.

EPD was used in only 11 of 55 cases (20\%). Angioplasties were performed in all 11 cases. In the EPD group, 9 of the 11 cases (81.8\%) were found to have severe wall ulcerations. All cases, except 1 , had severe stenosis with an average stenosis of $78.5 \%$ (Table 3 ).

Complications were divided into those occurring during the periprocedural period of 30 days and those on long-term follow-up. Only one case of minor stroke (EPD group) was observed in the entire cohort giving an overall stroke rate of $1.8 \%$ during the periprocedural period. No instance of myocardial infarction or death was reported during the periprocedural period. Also, no ipsilateral stroke or in-stent stenosis was reported during the follow-up period. There was no difference in outcome in those under 69 years of age or older than 70 . There was also no difference in age or cardiovascular risk factors. 


\section{Discussion}

Our data have revealed complication rates that are both comparable with other studies and are well within the ranges specified in the guidelines laid out by the American College of Cardiology Foundation (ACCF), the American Heart Association (AHA), and several other societies in the 2011 joint statement [12]. One incidence of mild stroke (1.8\%) was reported in the entire cohort, which is comparable to a number of studies $[4-6,15]$. This case of mild stroke had a lesion that was identified as short and contiguous with severe wall ulcerations. This patient, an 80-year-old woman with a prior middle cerebral artery stroke, had severe right carotid stenosis (90\%) extending from the carotid bulb to the proximal internal carotid artery. On angiography, the distal cervical/petrous internal carotid artery showed a sharp kink. No other abnormalities were picked and uneventful stenting was performed. Since the patient fulfilled the criteria set in the protocol (severe wall ulceration), EPD was used in this case. Postprocedure examination showed mild worsening of right proximal arm weakness that was assessed clinically, and later confirmed using noncontrast CAT scan imaging. The weakness was improving by the time the patient left the Angio suite. No end point outcome of ipsilateral stroke was reported after the 30-day periprocedural period. The CREST study reported a complications rate of $4.1 \%$ for periprocedural events, comparable to ours of $1.8 \%$ [4]. Another multicenter and multinational (Austria, Germany, and Switzerland) randomized trial, Stent-Protected Angioplasty versus Carotid Endarterectomy (SPACE), reported similar results [15]. During the SPACE trial, 1,200 symptomatic patients were assessed and the reported incidence of "ipsilateral ischemic stroke or death" after 30 days was $6.84 \%$ in patients randomized to CAS. Given this information from both large- and small-scale trials, carotid revascularization is considered to be safe and effective.

Use of EPD has essentially become universal to the stenting procedures worldwide with its use advocated in all cases. In all of the above trials, EPD has been vigorously practiced as referenced by its use in up to $96 \%$ of cases during the CREST trial [4, 5]. An extensive literature review has revealed the reasons for its widespread use, most important of which is a decrease in the incidence of 30-day combined stroke and death rate after CAS from 5.5\% without the use of EPD to $1.8 \%$ with EPD [7].

In our population, in all 11 patients where EPD was used, a conscious effort was made for its judicious use only in those who either had unstable plaque morphology or moderate to severe associated ulceration, potentially increasing the risk of embolism in these high-risk patients. With this approach, an effort was made to minimize vessel manipulation since undue or excessive maneuvering has been reportedly linked with an increased incidence of stroke or even stroke-like events that are clinically silent and not picked grossly. There is also evidence, as shown by Schlüter et al. [16], that neuroprotected CAS is associated with predominantly silent cerebral ischemia in approximately $25 \%$ of patients. Al-Mubarak et al. [17] also demonstrated that increased embolic events occurred due to endovascular manipulation by detection of increased microembolic signals recorded during and after the procedure using transcranial Doppler protocols. Another study by Men et al. [18] stated that in order to achieve a decreased number of Doppler-detected microembolic signals less catheter and guidewire manipulation should be practiced along with elimination of angioplasty both before and after stent placement. Additional drawbacks of EPD seen at an individual level, such as those reported by Cardaioli et al. [19], included severe vasospasms, and subsequent minor strokes. It is also shown by Maynar et al. [10] that the passage of EPD through the stenotic lesion causes a potential risk of dislodgment of unstable ulcerative plaques along with other complications such as failure in deployment or retrieval of EPD due to difficult anatomy and potential of emboli to escape during the retrieval process. Recently another randomized controlled trial conducted by Barbato et al. [20] assessed the role of cerebral protection in 36 cases of 
carotid stenting. These patients, randomized to either treatment, failed to show any significant difference in end point outcomes with or without the use of EPD. In fact, new MRIdetected lesions were seen in $72 \%$ of cases with EPD as compared to $44 \%$ of cases without EPD $(p=0.09)$. Similar results have recently been reported in the International Carotid Stenting Study whereby the use of cerebral protection did not modify the risk in patients [21]. One of the objectives of our study was to evaluate the safety and efficacy of the CAS procedure without the use of EPD. Our results of only one minor stroke $(1.8 \%)$ are in line with those reported by Maynar et al. [10] wherein the incidence of perioperative major stroke was 1\%, with one other minor stroke. No fatal major strokes or death were related to the procedure. This low incidence of minor stroke (1.8\%) compares favorably with those reported in other major trials including the study by Kastrup et al. [7] and most recently Binning et al. [11]. In the former, a rate of 1.8 and 5.5\% was reported for those treated with and without EPD, respectively. Based on these results, it is advocated that the use of EPD should be critically assessed before its adoption as a mandatory tool in the stenting procedure.

No significant association was established between the joint arterial characteristics and outcomes in the form of an adverse event. Similar results were reported by a study conducted by Doig et al. [22] in which 115 patients were assessed using DWI-MRI for cerebral lesions following the stenting procedure. During this study, carotid angiographic features failed to show significant results as a potential contributor to increased incidence of adverse events. Recently, a study conducted as a follow-up to the CREST trial, using the previously collected data, implicated angiographic features as a significant contributor towards the increased incidence of adverse events seen in the case of stenting [23]. The results indicated a strong relationship between the lesion length and stroke and death events. The incidence of stroke and death events in patients with longer lesion lengths $(>12.85 \mathrm{~mm})$ was 3.42 times higher in those who underwent CAS as compared to those having CEA (6.1 vs. 1.9\%, 95\% CI). Similar results were seen in case of sequential lesions with increased incidence in CAS as compared to CEA ( 5.8 vs. $0.7 \%, 95 \%$ CI). In our setup, angiographic features (except plaque morphology) played no role in either the use of EPD or the adverse events during or after the procedure.

During our study, all procedures were performed by a single operator with a 9-year experience in neurointervention (13.8 CAS procedures/year during the last 4 years). Standardization regarding operator experience is a factor lacking in many trials. Over the years, a number of efforts have been made in order to investigate the role of operator experience as a potential contributor towards the increased incidence of stroke events reported in some of the major randomized trials $[24,25]$. Using data from the Carotid Stenting Trialists' Collaboration from all 3 major randomized controlled trials (CREST, SPACE, and ICSS), Calvet et al. [24] performed a pooled analysis to account for the higher rates seen in these trials. Special considerations were made for operator experience in terms of operator lifetime CAS experience, operator stenting experience other than carotid stenting, and the annual in-trial operator volume. After reviewing 1,546 stenting cases, it was shown that patients who were treated by operators with low (mean $\leq 3.2$ procedures/year; risk $10.1 \%$; adjusted risk ratio $=2.30)$ and intermediate annual in-trial volume (3.2-5.6 procedures/year; $8.4 \%$; adjusted risk ratio $=1.93$ ) had a significantly higher risk of 30-day stroke or death compared to those treated by operators with high annual in-trial volume rate ( $>5.6$ procedures/year; $5.1 \%$ ). Recently, another study conducted amongst 24,701 Medicare beneficiaries who underwent CAS, assessed the role of operator experience in terms of median annual operator volume [25]. It was concluded that the mortality rates were higher amongst patients treated by very low-volume operators compared to patients treated by high-volume operators (adjusted odds ratio, 1.9; 95\% CI, 1.4-2.7; $p<0.001$ ). This shows that operator skills potentially influence the incidence of adverse events, and since a single operator performed all procedures in our setup, this helped add consistency to both the procedure and practice. 
EPD increases procedure cost which is an extremely important factor when it comes to providing health care in a developing country. Operator skills can effectively outweigh the feared complications indicated by angiographic arterial characteristics. Our results are comparable to those of previously reported major trials and are well within the complication thresholds suggested in current guidelines for both symptomatic and asymptomatic patients.

Limitations of this study include the retrospective nature of the data. Moreover, the sample size is relatively small, and a literature review reveals only a few existing studies addressing the issue of nonneuroprotected stenting and angioplasty. Although these findings are promising, a larger prospective cohort will need to be performed in order to validate our findings. In addition, an open-cell stent system was used in the majority of cases since closecell stents are still not available in our region.

\section{Conclusion}

Based on our single-center experience and findings of a relatively small sample size, carotid revascularization with stenting and angioplasty without EPD in experienced hands was found to be safe and efficacious in both symptomatic and asymptomatic carotid stenosis. The use of EPD should be justified and careful considerations, based on patient and angiographic profile, should be undertaken rather than a self-mandated approach, for it can lead to potential complications that can further worsen the outcome. In addition, limiting the use of unnecessary disposables lowers the procedure-related cost without compromising the patient outcome.

\section{Statement of Ethics}

Protocols for obtaining Ethics Certificate from the respective institutions were fulfilled along with acceptance of full responsibility, and compliance to maintaining patient confidentiality.

\section{Disclosure Statement}

The authors report that there is no conflict of interest to be disclosed. The authors do not have any personal, professional, or other affiliations with any products mentioned in the text.

\section{Funding Sources}

This research received no grant from any funding agency in the public, commercial or not-for-profit sectors.

\section{References}

1 Benjamin EJ, Blaha MJ, Chiuve SE, Cushman M, Das SR, Deo R, et al: Heart disease and stroke statistics - 2017 update: a report from the American Heart Association. Circulation 2017; 135:e146-e603.

2 Davis SM, Donnan GA: Carotid-artery stenting in stroke prevention. N Engl J Med 2010;363:80-82.

- 3 Rosamond W, Flegal K, Friday G, Furie K, Go A, Greenlund K, et al: Heart disease and stroke statistics - 2007 update. Circulation 2007;115:e69-e171.

-4 Brott TG, Hobson RW, Howard G, Roubin GS, Clark WM, Brooks W, et al: Stenting versus endarterectomy for treatment of carotid-artery stenosis. N Engl J Med 2010;363:11-23.

-5 Brott TG, Howard G, Roubin GS, Meschia JF, Mackey A, Brooks W, et al: Long-term results of stenting versus endarterectomy for carotid-artery stenosis. N Engl J Med 2016;374:1021-1031. 


\section{Interventional Neurology}

Bashirand Baig: Carotid Revascularization with and without EPD

6 Yadav JS, Wholey MH, Kuntz RE, Fayad P, Katzen BT, Mishkel GJ, et al: Protected carotid-artery stenting versus endarterectomy in high-risk patients. N Engl J Med 2004;351:1493-1501.

- 7 Kastrup A, Gröschel K, Krapf H, Brehm BR, Dichgans J, Schulz JB: Early outcome of carotid angioplasty and stenting with and without cerebral protection devices. Stroke 2003;34:813-819.

- 8 Castriota F, Cremonesi A, Manetti R, Liso A, Oshola K, Ricci E, et al: Impact of cerebral protection devices on early outcome of carotid stenting. J Endovasc Ther 2002;9:786-792.

9 Parodi JC, La Mura R, Ferreira LM, Mendez M V, Cersósimo H, Schönholz C, et al: Initial evaluation of carotid angioplasty and stenting with three different cerebral protection devices. J Vasc Surg 2000;32:1127-1136.

-10 Maynar M, Baldi S, Rostagno R, Zander T, Rabellino M, Llorens R, et al: Carotid stenting without use of balloon angioplasty and distal protection devices: preliminary experience in 100 cases. Am J Neuroradiol 2007;28: 1378-1383.

11 Binning MJ, Maxwell CR, Stofko D, Zerr M, Maghazehe K, Liebman K, et al: Carotid artery angioplasty and stenting without distal embolic protection devices. Neurosurgery 2017;80:60-64.

-12 Brott TG, Halperin JL, Abbara S, Bacharach JM, Barr JD, Bush RL, et al: 2011 ASA/ACCF/AHA/AANN/AANS/ ACR/ASNR/CNS/SAIP/SCAI/SIR/SNIS/SVM/SVS guideline on the management of patients with extracranial carotid and vertebral artery disease: executive summary. Circulation 2011;124:489-532.

13 Lovett JK, Gallagher PJ, Hands LJ, Walton J, Rothwell PM: Histological correlates of carotid plaque surface morphology on lumen contrast imaging. Circulation 2004;110:2190-2197.

- 14 Ferguson GG, Eliasziw M, Barr HWK, Clagett GP, Barnes RW, Wallace MC, et al: The North American Symptomatic Carotid Endarterectomy Trial. Stroke 1999;30:1751-1758.

-15 Wiesmann M, Schöpf V, Jansen O, Brückmann H: Stent-protected angioplasty versus carotid endarterectomy in patients with carotid artery stenosis: meta-analysis of randomized trial data. Eur Radiol 2008;18:29562966.

-16 Schlüter M, Tübler T, Steffens JC, Mathey DG, Schofer J: Focal ischemia of the brain after neuroprotected carotid artery stenting. J Am Coll Cardiol 2003;42:1007-1013.

-17 Al-Mubarak N, Roubin GS, Vitek JJ, Iyer SS, New G, Leon MB: Effect of the distal-balloon protection system on microembolization during carotid stenting. Circulation 2001;104:1999-2002.

18 Men S, Lownie SP, Pelz DM: Carotid stenting without angioplasty. Can J Neurol Sci 2002;29:175-179.

-19 Cardaioli P, Giordan M, Panfili M, Chioin R: Complication with an embolic protection device during carotid angioplasty. Catheter Cardiovasc Interv 2004;62:234-236.

20 Barbato JE, Dillavou E, Horowitz MB, Jovin TG, Kanal E, David S, et al: A randomized trial of carotid artery stenting with and without cerebral protection. J Vasc Surg 2018;47:760-765.

21 Featherstone RL, Dobson J, Ederle J, Doig D, Bonati LH, Morris S, et al: Carotid artery stenting compared with endarterectomy in patients with symptomatic carotid stenosis (International Carotid Stenting Study): a randomised controlled trial with cost-effectiveness analysis. Health Technol Assess 2016;20:1-94.

22 Doig D, Hobson BM, Müller M, Jäger HR, Featherstone RL, Brown MM, et al: Carotid anatomy does not predict the risk of new ischaemic brain lesions on diffusion-weighted imaging after carotid artery stenting in the ICSS-MRI substudy. Eur J Vasc Endovasc Surg 2016;51:14-20.

-23 Moore WS, Popma JJ, Roubin GS, Voeks JH, Cutlip DE, Jones M, et al: Carotid angiographic characteristics in the CREST trial were major contributors to periprocedural stroke and death differences between carotid artery stenting and carotid endarterectomy. J Vasc Surg 2018;63:851-858.e1.

24 Calvet D, Mas J-L, Algra A, Becquemin J-P, Bonati LH, Dobson J, et al: Carotid stenting. Stroke 2014;45:527-532.

-25 Nallamothu BK, Gurm HS, Ting HH, Goodney PP, Rogers MAM, Curtis JP, et al: Operator experience and outcomes with carotid stenting in medicare beneficiaries. JAMA 2011;306:1338-1343. 